\title{
An EOQ Model with Multiple Suppliers and Random Capacity
}

\author{
Aslı Sencer Erdem, ${ }^{1}$ Mehmet Murat Fadılog̃lu, ${ }^{2}$ Süleyman Özekici ${ }^{3}$ \\ ${ }^{1}$ Bog̃aziçi University, Department of Management Information Systems, 34342 Bebek, İstanbul, Turkey \\ ${ }^{2}$ Bilkent University, Department of Industrial Engineering, 06800 Bilkent, Ankara, Turkey \\ ${ }^{3}$ Koç University, Department of Industrial Engineering, 34450 Sarlyer, İstanbul, Turkey
}

Received 17 June 2004; revised 15 July 2005; accepted 10 August 2005

DOI 10.1002/nav.20125

Published online 7 December 2005 in Wiley InterScience (www.interscience.wiley.com).

\begin{abstract}
We consider an EOQ model with multiple suppliers that have random capacities, which leads to uncertain yield in orders. A given order is fully received from a supplier if the order quantity is less than the supplier's capacity; otherwise, the quantity received is equal to the available capacity. The optimal order quantities for the suppliers can be obtained as the unique solution of an implicit set of equations in which the expected unsatisfied order is the same for each supplier. Further characterizations and properties are obtained for the uniform and exponential capacity cases with discussions on the issues related to diversification among suppliers. (C) 2005 Wiley Periodicals, Inc. Naval Research Logistics 53: 101-114, 2006.
\end{abstract}

Keywords: EOQ; random capacity; multiple suppliers; order diversification

\section{INTRODUCTION}

Continuous-review inventory systems with random yield have been modeled in several different ways in the literature. The original idea behind yield randomness is due to the fact that the quantity received from the supplier may differ somewhat from the quantity ordered. As discussed in the review by Yano and Lee [20], a common way to model yield uncertainty is to take the random yield $Y_{q}$ "stochastically proportional" to the order quantity $q$ so that $Y_{q}=U q$. Here, $U$ is a random variable that may represent, for example, the fraction of non-defective items. Earlier examples of these models can be found in Karlin [11], Silver [17], and Shih [16]. Lee and Yano [12] formulate the multistage serial production system with random yield and deterministic demand. Henig and Gerchak [10] provide a comprehensive analysis of general periodic-review models with random yield in multi-periods and show the optimality of "nonorder-up-to" policies. Unfortunately, these policies are not as simple as the well-known base-stock and $(s, S)$ policies. Under such polices, no order is given if inventory position is over a critical threshold, but the order quantity below this level does not necessarily bring

Correspondence to: A. S. Erdem (asli.erdem@boun.edu.tr); M. M. Fadılog̃lu (mmurat@bilkent.edu.tr); S. Özekici (sozekici@ ku.edu.tr) the inventory position to a fixed base-stock level. Parlar and Berkin [14] and Gürler and Parlar [9] analyze the case where supply is available only during intervals of random length. Özekici and Parlar [13] introduce the idea of a random environment that affects the demand, supply, and all cost parameters. They show the optimality of environment-dependent base-stock and $(s, S)$ policies when the supplier is unreliable.

Another approach in modeling random yield is to treat yield uncertainty as a consequence of random capacity. This may be due to unreliable machinery and unplanned maintenance in a production system or possibly finite availability of items in an inventory system. In these models, the quantity that is actually received is $Y_{q}=\min \{q, A\}$ if $q$ is the order quantity and $A$ is the random capacity. Ciarallo, Akella, and Morton [2], for example, propose a periodic-review production model with random capacity where the base-stock policy is found to be optimal. Wang and Gerchak [18] further extend this model to allow random capacity and random yield simultaneously, i.e., $Y_{q}=$ $U \min \{q, A\}$. The structure of the optimal ordering policy in each period is similar to that of Henig and Gerchak [10]; i.e., an order is given if the inventory level at the beginning of the period is below a critical point; otherwise, no order is given. Güllü [8] considers a model where the yield depends on the quantity present at the supplier in addition to the availability of the supplier. Erdem and 
Özekici [5] analyze periodic models with random capacity in a random environment and show the optimality of environment-dependent base-stock policies when there is no fixed cost. In continuous-review environments, Wang and Gerchak [19] analyze the effects of variable capacity on optimal lot size and obtain optimality conditions for generally distributed capacity.

An important factor that is missing or neglected in the literature is the necessity to include multiple suppliers in random capacity models. Random supplier capacity directly implies that orders should be diversified to many suppliers in order to reduce the risk associated with insufficient capacity of the suppliers. In practice, the assumption that there is a single supplier is often false and unrealistic since variability of actual yield can be reduced through diversification of the risk by working with a number of suppliers. Extensive studies have been done on the random yield and random capacity models with a single supplier. On the other hand, substantially less effort has been spent on models with multiple suppliers due mainly to their apparent complexity. In the original work is by Anupindi and Akella [1] they consider the single-period problem of ordering from two different suppliers with stochastically proportional yields. The optimal policy, which is determined by two critical order points, is of the form: "order from both," "order from the cheaper supplier," or "do not order." The issue of order diversification is discussed by Erdem [4] in a single-period model where there are two suppliers with random capacities to show that the total order quantity does not necessarily bring the inventory position to a base-stock level. Even with no fixed cost of ordering, the optimal policy may be rather complicated. In a continuous-review system, diversification under yield randomness was first analyzed in the EOQ context by Gerchak and Parlar [7] where two suppliers with identical cost parameters and nonidentical stochastically proportional yields are considered. Parlar and Wang [15] extended these results to supplier-specific unit cost of ordering and found the optimal order quantities explicitly. All these multiple supplier studies concentrate on two supplier models. The only other study that considers an unlimited number of suppliers like our study is by Fadıloğlu, Berk, and Gürbüz [6]. They analyze the multiple supplier binomial yield problem in an EOQ setting and show that diversification is not always preferable.

In this paper, we discuss issues related to random capacity and multiple suppliers in the well-known EOQ model. Our problem is similar to that of Parlar and Wang [15] since we both discuss yield uncertainty and multiple suppliers in the EOQ model, but the yield structures are substantially different and the number of suppliers is not limited to two. Another closely related study is by Wang and Gerchak [19] where there is a single supplier with random capacity. We generalize these models to allow multiple nonidentical suppliers with random capacities in a continuous-review system.
The organization of the paper is as follows: In Section 2, we derive the general characterization for the optimal order quantities of the random capacity EOQ model with multiple suppliers. Sections 3 and 4 are devoted to the special cases of the uniform and exponential capacity suppliers where some interesting properties of the optimal solution are provided. In Section 5, the issues related to diversification among suppliers are discussed and results are demonstrated by some numerical illustrations. The reader should refer to the Appendix for the lengthy proofs.

\section{EOQ MODEL WITH MULTIPLE SUPPLIERS}

If it is certain that the suppliers deliver what is ordered, then it is surely most economical to work with the supplier who provides the product at the least cost for the desired quality level. However, this is often not the case in real life and it is a fact that usually companies prefer to work with more than one supplier. Under random supplier capacities, the retailer should order from a number of suppliers in order to diversify the risk associated with shortages.

In this section, we model the setting described above by assuming that there are $n$ suppliers with constant lead times. Joint orders are given to $n$ suppliers with independent random capacities $\left\{A_{i} ; i=1,2, \ldots, n\right\}$ having distribution functions $F_{i}$ and density $f_{i}$. Let $q_{i}$ be the order quantity for the $i$ th supplier; then the amount received from supplier $i$ is $Y_{q_{i}}=\min \left\{q_{i}, A_{i}\right\}$. At the beginning of any order cycle, if $q_{1}, q_{2}, \ldots, q_{n}$ are the order quantities for the $n$ suppliers, then the total amount actually received from the joint order is $Y_{q_{1}}+Y_{q_{2}}+\cdots+Y_{q_{n}}$. We assume that $\bar{F}_{i}(x)>0$ for all $x \geq 0$ unless stated otherwise throughout the remainder of the paper. However, we may relax this assumption and analyze the model when there is an upper bound $a_{i}$ for the capacity of the supplier $i$, such that $\bar{F}_{i}(x)=0$ for all $x \geq a_{i}$. This is illustrated in the uniform capacities setting in Section 3.

Our model assumes that all the available suppliers are used. The joint order (setup) cost is $K_{n}$, the cost of giving an order to all of the $n$ available suppliers. If which subset of the suppliers used is also a decision, then the order cost would be a function of the subset that would be the sum of a supplier specific (minor) setup and an order specific (major) setup. This situation is discussed and illustrated in Section 5.3.

The unit purchase cost is $c$ and unit inventory holding cost is $h$ per unit time. We suppose that all suppliers offer the same unit price since we want to focus on the effect of random capacity on the ordering policy.

Using renewal theory, the long run average total cost function is simply the ratio of expected total cost per cycle to expected cycle length. The required expression for the average cost as a function of the order quantities for the $n$ suppliers is 


$$
\frac{K_{n}+c E\left[Y_{q_{1}}+Y_{q_{2}}+\cdots+Y_{q_{n}}\right]+h E\left[\left(Y_{q_{1}}+Y_{q_{2}}+\cdots+Y_{q_{n}}\right)^{2}\right] /(2 D)}{E\left[Y_{q_{1}}+Y_{q_{2}}+\cdots+Y_{q_{n}}\right] / D} .
$$

The numerator of (1) is the sum of joint ordering cost, expected cost of purchasing, and the expected total inventory holding cost in a cycle. Now, we derive an expression for $E\left[\left(Y_{q_{1}}+Y_{q_{2}}+\cdots+Y_{q_{n}}\right)^{2}\right]$ that will simplify (1). Since the random capacities are assumed to be independent, we have

$$
\begin{aligned}
E\left[\left(Y_{q_{1}}+Y_{q_{2}}+\right.\right. & \left.\left.\cdots+Y_{q_{n}}\right)^{2}\right] \\
& =\sum_{i=1}^{n} E\left[Y_{q_{i}}^{2}\right]+\sum_{i, j=1, j \neq i}^{n} E\left[Y_{q_{i}}\right] E\left[Y_{q_{j}}\right] .
\end{aligned}
$$

The average total cost expression in (1) can now be rewritten as

$c D$

$$
+\frac{K_{n} D+h\left[\sum_{i=1}^{n} E\left[Y_{q_{i}}^{2}\right]+\sum_{i, j=1, j \neq i}^{n} E\left[Y_{q_{i}}\right] E\left[Y_{q_{j}}\right]\right] / 2}{\sum_{i=1}^{n} E\left[Y_{q_{i}}\right]} .
$$

The total purchase cost $c D$ can be disregarded in the optimization problem so that it now becomes $\min _{q_{1}, q_{2}, \ldots, q_{n} \geq 0}$ $T C\left(q_{1}, q_{2}, \ldots, q_{n}\right)$, where

$$
\begin{aligned}
& T C\left(q_{1}, q_{2}, \ldots, q_{n}\right) \\
& =\frac{K_{n} D+h\left[\sum_{i=1}^{n} E\left[Y_{q_{i}}^{2}\right]+\sum_{i, j=1, j \neq i}^{n} E\left[Y_{q_{i}}\right] E\left[Y_{q_{j}}\right]\right] / 2}{\sum_{i=1}^{n} E\left[Y_{q_{i}}\right]} .
\end{aligned}
$$

At this point, we need to provide the expressions for $E\left[Y_{q_{i}}\right]$ and $E\left[Y_{q_{i}}^{2}\right]$ in (4). We can compute $E\left[Y_{q_{i}}\right]$ as

$$
\begin{aligned}
& E\left[Y_{q_{i}}\right]=\int_{0}^{+\infty} \min \left\{q_{i}, y\right\} d F_{i}(y) \\
&=\int_{0}^{q_{i}} y d F_{i}(y)+q_{i} \bar{F}_{i}\left(q_{i}\right) .
\end{aligned}
$$

Note that $E\left[Y_{q_{i}}\right] \leq E\left[A_{i}\right]$ with $\lim _{q_{i} \rightarrow+\infty} E\left[Y_{q_{i}}\right]=E\left[A_{i}\right]$. The derivative of $E\left[Y_{q_{i}}\right]$ with respect to order quantity $q_{i}$ is nonnegative since

$$
\frac{d E\left[Y_{q_{i}}\right]}{d q_{i}}=\int_{q_{i}}^{+\infty} d F_{i}(y)=\bar{F}_{i}\left(q_{i}\right) \geq 0,
$$

while the second derivative is nonpositive since

$$
\frac{d^{2} E\left[Y_{q_{i}}\right]}{d q_{i}^{2}}=-f_{i}\left(q_{i}\right) \leq 0 .
$$

We conclude that $E\left[Y_{q_{i}}\right]$ is a concave increasing function with $\left.E\left[Y_{q_{i}}\right]\right|_{q_{i}=0}=0$ and it converges to $E\left[A_{i}\right]$ as $q_{i}$ increases.
Similarly, we obtain $E\left[Y_{q_{i}}^{2}\right]$ by using $Y_{q_{i}}=\min \left\{q_{i}, A_{i}\right\}$ as

$$
\begin{aligned}
& E\left[Y_{q_{i}}^{2}\right]=\int_{0}^{+\infty} \min \left\{q_{i}, y\right\}^{2} d F_{i}(y) \\
&=\int_{0}^{q_{i}} y^{2} d F_{i}(y)+q_{i}^{2} \bar{F}_{i}\left(q_{i}\right) .
\end{aligned}
$$

Now, $E\left[Y_{q_{i}}^{2}\right] \leq E\left[A_{i}^{2}\right]$ with $\lim _{q_{i} \rightarrow+\infty} E\left[Y_{q_{i}}^{2}\right]=E\left[A_{i}^{2}\right]$. Furthermore, we differentiate $E\left[Y_{q_{i}}^{2}\right]$ in (8) with respect to $q_{i}$ so that

$$
\frac{d E\left[Y_{q_{i}}^{2}\right]}{d q_{i}}=2 q_{i} \int_{q_{i}}^{+\infty} d F_{i}\left(y_{i}\right)=2 q_{i} \bar{F}\left(q_{i}\right) \geq 0
$$

and find that $E\left[Y_{q_{i}}^{2}\right]$ is also an increasing function with $\left.E\left[Y_{q_{i}}^{2}\right]\right|_{q_{i}=0}=0$ and converging to $E\left[A_{i}^{2}\right]$ as $q_{i}$ increases. However, it is not necessarily concave.

We can now get into the details of solving our optimization problem to minimize $T C\left(q_{1}, q_{2}, \ldots, q_{n}\right)$. The following result provides an interesting implicit characterization for the optimal solution of the random capacity EOQ model with multiple suppliers.

THEOREM 1: The optimal order quantities of the random capacity EOQ model with $n$ suppliers are given as the unique nonnegative solution of the following set of equations:

$$
\begin{array}{r}
2 q_{1} \sum_{i=1}^{n} E\left[Y_{q_{i}}\right]+2 \sum_{i=2}^{n} \sum_{j=i}^{n} E\left[Y_{q_{i}}\right] E\left[Y_{q_{j}}\right] \\
-\sum_{i=1}^{n} E\left[Y_{q_{i}}^{2}\right]=\frac{2 K_{n} D}{h} \\
q_{1}-E\left[Y_{q_{1}}\right]=q_{2}-E\left[Y_{q_{2}}\right]=\cdots=q_{n}-E\left[Y_{q_{n}}\right] .
\end{array}
$$

Theorem 1 reveals an important property of the optimal order quantities of the random capacity EOQ model with multiple suppliers. If we reconsider the condition in (11), we see that $q_{i}-E\left[Y_{q_{i}}\right]$ is a positive constant since $Y_{q_{i}} \leq q_{i}$ for all $q_{i}$. This optimal policy stipulates that the retailer's order should be diversified such that the expected number of unfulfilled order units is the same for each supplier. This relation specifies the optimal order quantities from all suppliers as a function of the optimal order quantity from any given supplier. Since $q_{i}-E\left[Y_{q_{i}}\right]$ is an increasing function of $q_{i}$, if the order quantity from any supplier increases, then all other order quantities should also increase in order to satisfy (11). It is (10) that determines what the optimal order quantity should be for the first supplier and thereby establishes the optimal order quantities for the other suppliers as well. We see in (10) that as $2 K_{n} D / h$, the square of classical EOQ formula, increases, all the order quantities increase. 
If the setup cost is not dependent on the number of suppliers, it is always better to diversify among all available suppliers since setting any of the order quantities to zero would necessitate setting all order quantities to zero so that (10) is satisfied. This is quite intuitive, since by diversifying our orders we decrease the probability that we exceed the capacities of the suppliers and thereby decrease the uncertainty in what we receive.

In the next corollary we show that the optimal order quantities from the suppliers follow the stochastic order of their capacity distributions. The corollary states that if the probability of fully receiving the order is greater for a supplier compared to another, then we should order more from this supplier.

\section{COROLLARY 2: Let $A_{i} \succeq_{s t} A_{j}$ then $q_{i}^{*} \geq q_{j}^{*}$.}

PROOF: By definition, if $A_{i} \succeq_{s t} A_{j}$, then $\bar{F}_{i}(u) \geq \bar{F}_{j}(u)$ for all $u \geq 0$. Since $A_{i} \succeq_{s t} A_{j}$ implies that $\operatorname{Min}\left(A_{i}, q\right) \succeq_{s t}$ $\operatorname{Min}\left(A_{j}, q\right)$, we can write $E\left[\operatorname{Min}\left(A_{i}, q\right)\right] \geq E\left[\operatorname{Min}\left(A_{j}, q\right)\right]$ or simply $\left.E\left[Y_{q_{i}}\right]\right|_{q_{i}=q} \geq\left. E\left[Y_{q_{j}}\right]\right|_{q j=q}$. Since $q_{k}-\left.E\left[Y_{q_{k}}\right]\right|_{q_{k}=q}$ is a non-decreasing function of $q$ for any supplier $k, q_{i}^{*} \geq q_{j}^{*}$ so that the optimality condition (11) is satisfied.

We should remark that the underlying assumption behind the theory presented in this section is that the random capacities of the suppliers are unbounded. When bounded capacities are considered, the system of equations given in Theorem 1 may not yield a solution. This is due to the fact that even if the retailer orders more than the capacity bound from a supplier, the distribution of the quantity received will be the same as when the capacity bound is ordered. Thus, $q_{i}-E\left[Y_{q_{i}}\right]$ will be constant after the bound is reached for supplier $i$. This means that it may be impossible to set the expected number of unfulfilled order units to the level satisfying (11). Then, the order quantity for that supplier should be set to its capacity bound, and the optimization problem should be solved for the rest of the supplier order quantities. Yet, one should note that Corollary 2 is always valid irrespective of the boundedness of the capacity distributions. The approach in the case of multiple suppliers with bounded capacities is illustrated in the next section.

\section{SUPPLIERS WITH UNIFORM CAPACITIES}

In real life, the suppliers have always a bound on their capacity, which means that they can never satisfy an infinite order. Thus, it is practically relevant to consider bounded capacity distributions. Among the bounded distributions, the uniform distribution is the simplest-yet reasonabledistribution to model the random capacity. The underlying assumption while using the uniform capacity distribution is that any capacity value within a given interval is equally likely. In the case of lack of knowledge about the true capacity distribution, the uniform distribution is the best choice from a practical point of view. Furthermore, noting that the supplier may be unable to send any quantity from time to time due to production shutdowns, etc., one can claim that the lower bound on the capacity distribution has to be zero.

When the random capacity of a supplier is uniformly distributed on $[0, a]$ for some $a>0$, by using (5) and (8) we can easily obtain

$$
E\left[Y_{q}\right]=q-\frac{q^{2}}{2 a}=q\left(1-\frac{q}{2 a}\right)
$$

and

$$
E\left[Y_{q}^{2}\right]=q^{2}-\frac{2}{3 a} q^{3}=q^{2}\left(1-\frac{2 q}{3 a}\right) .
$$

In the single supplier case with $n=1$, the optimality condition (10) yields

$$
w(q)=q^{2}\left(1-\frac{q}{3 a}\right)=\frac{2 K D}{h} .
$$

for $0 \leq q \leq a$. It is not surprising at all that $\lim _{a \rightarrow+\infty} q^{2}(1-$ $\left.\frac{q}{3 a}\right)=q^{2}$, leading to the classical EOQ model. One can show that $w(q)$ is strictly increasing on $[0, a]$ with $w(0)=0$ and $w(a)=2 a^{2} / 3$. Therefore, the optimal order quantity is the unique solution of (14) in $[0, a]$ if $a^{2} \geq 3 K D / h$. However, if $a^{2}<3 K D / h$, then the optimal order quantity is $a$ since it does not make sense to order more than what the supplier can possibly deliver.

Note that our assumption $\bar{F}(x)>0$ is clearly not satisfied in the uniform capacity case since $\bar{F}(x)=0$ whenever $x \geq a$. Therefore, it is no longer true that the optimal order quantity is the unique solution of (14). However, this does not constitute a major obstacle. It suffices to treat this problem as a constrained optimization problem with $0 \leq q \leq a$ so that whenever there is no solution of (14) on $[0, a]$ the optimal order quantity is $a$.

We now consider the general case with $n$ suppliers where the capacity $A_{i}$ of supplier $i$ is uniformly distributed between zero and some $a_{i}>0$. In this section, we suggest a procedure to find the optimal order quantities when the random capacities have upper bounds.

THEOREM 3: Consider the EOQ model with $n$ suppliers that have uniform capacities so that $A_{i} \sim$ Uniform $\left[0, a_{i}\right]$ for some $a_{i}>0$ and $i=1,2, \ldots, n$. Let $q_{i}^{0}$ be a solution of the equations

$$
\begin{aligned}
& {\left[\frac{n(n-1)}{4 a_{1}^{2}}\right] q_{1}^{4}+\left[\frac{2-3 n}{3 a_{1}} \sum_{i=1}^{n} \sqrt{\frac{a_{i}}{a_{1}}}\right] q_{1}^{3}} \\
& +\left[2 \sum_{i=1}^{n} \sum_{j=i}^{n} \sqrt{\frac{a_{i}}{a_{1}} \frac{a_{j}}{a_{1}}}-\sum_{i=1}^{n} \frac{a_{i}}{a_{1}}\right] q_{1}^{2}=\frac{2 K_{n} D}{h}
\end{aligned}
$$


and

$$
q_{k}=\sqrt{\frac{a_{k}}{a_{1}}} q_{1}
$$

for $k=2,3, \ldots, n$. If $q_{i}^{0} \leq a_{i}$ for all $i=1,2, \ldots, n$, then the optimal order quantity is $Q_{i}=q_{i}^{0}$ for all $i$.

Theorem 3 states that, if all the order quantities given by the first order conditions do not exceed the capacity bounds, then the optimal order quantities are uniquely determined. Otherwise, the optimal order quantity for that supplier must be equal to its capacity bound since there is no point in ordering more. In Corollary 4, we state a related property of the optimal solution if the first order conditions do not lead to a feasible solution. It is interesting that the optimality condition is a cubic equation for $n=1$ and a quartic equation for any larger $n$ as stated in (14) and (15).

COROLLARY 4: Suppose without loss of generality that the suppliers are ordered such that $a_{1} \geq a_{2} \geq \cdots \geq a_{n}$. If $k=\max \left\{i ; q_{i}^{0}<a_{i}, i=1,2, \ldots, n\right\}$, then $Q_{i}=a_{i}$ for all $i=k+1, k+2, \ldots, n$.

PROOF: It is sufficient to show that if $q_{i}^{0}>a_{i}$, then $q_{i+1}^{0}>$ $a_{i+1}$. Using (16), we can write

$$
q_{i+1}^{0}=\sqrt{\frac{a_{i+1}}{a_{1}}} q_{1}^{0}=\sqrt{\frac{a_{i+1}}{a_{i}}} \sqrt{\frac{a_{i}}{a_{1}}} q_{1}^{0}=\sqrt{\frac{a_{i+1}}{a_{i}}} q_{i}^{0} .
$$

Since $a_{i} \geq a_{i+1}, \sqrt{a_{i+1} / a_{i}} \geq a_{i+1} / a_{i}$. Also noting that $q_{i}^{0}>$ $a_{i}$, (17) leads to

$$
q_{i+1}^{0} \geq \frac{a_{i+1}}{a_{i}} q_{i}^{0}>\frac{a_{i+1}}{a_{i}} a_{i}=a_{i+1} .
$$

Thus, $q_{i+1}^{0}>a_{i+1}$ and the rest follows by induction on $i$.
By Corollary 4, if the order quantities determined by the first order conditions in Theorem 3 are less than or equal to the capacity bounds for the first $k$ high-capacity suppliers, then the optimal order quantities for all the other suppliers are at their capacity bounds, i.e., $Q_{i}=a_{i}$ since $q_{i}^{0} \geq a_{i}$ for $i=$ $k+1, \ldots, n$. So, by setting $Q_{k+1}=a_{k+1}, \ldots, Q_{n}=a_{n}$, the first order conditions defined by (10) must be resolved to find the optimal order quantities $Q_{1}, Q_{2}, \ldots, Q_{k}$. This implies that we need to replace (15) by

$$
\begin{aligned}
& {\left[\frac{k(k-1)}{4 a_{1}^{2}}\right] q_{1}^{4}+\left[\frac{2-3 k}{3 a_{1}} \sum_{i=1}^{k} \sqrt{\frac{a_{i}}{a_{1}}}\right] q_{1}^{3}} \\
& +\left[2 \sum_{i=1}^{k} \sum_{j=i}^{k} \sqrt{\frac{a_{i}}{a_{1}} \frac{a_{j}}{a_{1}}}-\sum_{i=1}^{k} \frac{a_{i}}{a_{1}}\right] q_{1}^{2}=\frac{2 K_{n} D}{h} .
\end{aligned}
$$

Once $Q_{1}$ is found by using (19), $Q_{2}, \ldots, Q_{k}$ are determined by using the first order conditions in (16). Let us note that this will lead to an increase in the previously obtained order quantities $q_{1}^{0}, q_{2}^{0}, \ldots, q_{k}^{0}$ found by using Theorem 3 and this iterative procedure is repeated until $Q_{i} \leq a_{i}$ for all $i$.

In Table 1 we provide some numerical illustrations for the two suppliers problem with uniform capacities. Experiments are made in two sets for $a_{1}=85$ and $a_{1}=100$, respectively, and the corresponding $a_{2}$ levels are chosen such that $a_{1} \geq a_{2}$. The cost parameters are $K=200, D=32, h=2$.

As a consequence of Corollary 4 , if the higher capacity supplier is ordered at its capacity bound, (i.e., $Q_{1}=a_{1}$ ), then so is the second supplier. It is also easy to check that the expected number of unfulfilled order units from both suppliers $Q_{i}-E\left[Y_{Q_{i}}\right]$ is the same if both order quantities are lower than the capacity bounds. If the order quantity is at capacity bound, then $E\left[Y_{Q_{i}}\right]=E\left[A_{i}\right]=a_{i} / 2$. Finally, if the capacity constraints are more restrictive due to low levels of $a_{2}$, then

Table 1. Numerical results of the two suppliers problem with uniform capacities.

\begin{tabular}{rrrrcccrr}
\hline$a_{1}$ & $a_{2}$ & $Q_{1}$ & \multicolumn{1}{c}{$Q_{2}$} & $Q_{1}-E\left[Y_{Q_{1}}\right]$ & $Q_{2}-E\left[Y_{Q_{2}}\right]$ & $E\left[Y_{Q_{1}}\right]$ & $E\left[Y_{Q_{2}}\right]$ & $T C$ \\
\hline 85 & 85 & 49.44 & 49.44 & 14.38 & 14.38 & 35.06 & 35.06 & 169.01 \\
& 50 & 61.86 & 47.45 & 22.51 & 2.51 & 39.35 & 24.93 & 173.19 \\
& 30 & 75.26 & 30.00 & 32.32 & 15.00 & 41.94 & 15.00 & 180.52 \\
& 25 & 85.00 & 25.00 & 42.50 & 12.50 & 42.50 & 12.50 & 183.26 \\
& 10 & 85.00 & 10.00 & 42.50 & 5.00 & 42.50 & 5.00 & 195.09 \\
& 5 & 85.00 & 5.00 & 42.50 & 2.50 & 42.50 & 2.50 & 200.65 \\
& 2 & 85.00 & 2.00 & 42.50 & 1.00 & 42.50 & 1.00 & 204.48 \\
100 & 100 & 47.40 & 47.40 & 11.23 & 11.23 & 36.17 & 36.17 & 167.14 \\
& 50 & 61.21 & 43.28 & 18.73 & 18.73 & 42.48 & 24.55 & 171.51 \\
& 30 & 73.23 & 30.00 & 26.81 & 15.00 & 46.42 & 15.00 & 176.45 \\
& 20 & 80.34 & 20.00 & 32.28 & 10.00 & 48.07 & 10.00 & 180.69 \\
& 10 & 100.00 & 10.00 & 50.00 & 5.00 & 50.00 & 5.00 & 186.67 \\
& 5 & 100.00 & 5.00 & 50.00 & 2.50 & 50.00 & 2.50 & 190.32 \\
& 2 & 100.00 & 2.00 & 50.00 & 1.00 & 50.00 & 1.00 & 192.84 \\
\hline
\end{tabular}


the optimal expected total cost is increased, which is intuitive as well.

These illustrations help us provide some intuition for the multiple suppliers problem with bounded capacities: If the optimal order quantities for certain suppliers are at their bounds, then there is a capacity problem at these suppliers that prevents us from diversifying our orders as in the absence of bounds. Obviously, this leads to an increase in the average costs. We can overcome this difficulty by increasing the number of suppliers we are working with.

\section{SUPPLIERS WITH EXPONENTIAL CAPACITIES}

In this section, we assume that the random capacities are exponentially distributed with parameter $\mu$ so that $F(x)=$ $1-\exp (-\mu x), x \geq 0$. Exponential capacities provide us an ideal setting for getting insight about unbounded capacity case since it is computationally more tractable compared to other unbounded distributions. Specifically, the exponential case yields some explicit solutions. The analysis of the exponential capacities provided in this section is then used for an illustration of the unbounded capacities case in contrast with the bounded capacities case, discussed in the previous section.

By using (5) and (8) one can easily show that

$$
E\left[Y_{q}\right]=\frac{1}{\mu}\left(1-e^{-\mu q}\right)
$$

and

$$
E\left[Y_{q}^{2}\right]=\frac{2}{\mu^{2}}\left(1-(1+\mu q) e^{-\mu q}\right)
$$

In the single supplier case with $n=1$, the optimal order quantity satisfies (10) as

$$
\frac{2\left(\mu Q+e^{-\mu Q}-1\right)}{\mu^{2}}=\frac{2 K D}{h} .
$$

It is not surprising at all that $\lim _{\mu \rightarrow 0} \frac{2\left(\mu Q+e^{-\mu Q}-1\right)}{\mu^{2}}=Q^{2}$, leading to the classical EOQ model. As a matter of fact, it is not difficult to show that, as $\mu$ gets smaller, the random capacity gets stochastically larger and the order quantity gets smaller.

The solution of (22) is given explicitly by

$$
Q=\frac{1}{\mu}\left[1+\hat{K} \mu^{2}+W\left(-e^{-\left(1+\hat{K} \mu^{2}\right)}\right)\right],
$$

where $\hat{K}=K D / h$ for notational simplicity and $W$ is the Lambert $W$ function that can be computed with arbitrary precision. The Lambert $W$ function is the inverse of $f(y)=y e^{y}$ so that it satisfies $W(x) e^{W(x)}=x$ for any $x$. It arises naturally in some interesting problems and has some nice properties. The reader is referred to Corless et al. [3] for details on Lambert $W$ function. In our case, since $0 \leq \hat{K} \leq+\infty$, we have $-e^{-1} \leq-e^{-\left(1+\hat{K} \mu^{2}\right)} \leq 0$ and

$$
-1=W\left(-e^{-1}\right) \leq W\left(-e^{-\left(1+\hat{K} \mu^{2}\right)}\right) \leq W(0)=0
$$

so that

$$
\hat{K} \mu \leq Q \leq \frac{1}{\mu}+\hat{K} \mu
$$

Using (22) and (25) for small and large values of $\mu$, we can use the approximation

$$
Q \cong \begin{cases}K D \mu / h, & \text { for large } \mu \\ \sqrt{2 K D / h} & \text { for small } \mu\end{cases}
$$

without having to use the Lambert $W$ function. Using the fact that $Q \geq E O Q$ (Wang and Gerchak [19]) and (25), we can obtain bounds $L$ and $U$ for $Q$ such that

$$
L=\max \left\{\sqrt{\frac{2 K D}{h}}, \frac{K D \mu}{h}\right\} \leq Q \leq \frac{1}{\mu}+\frac{K D \mu}{h}=U .
$$

We can rewrite the bounds as

$$
[L, U]=\left[\sqrt{\frac{2 K D}{h}} \max \left\{1, \sqrt{\frac{\hat{K}}{2}} \mu\right\}, \frac{1}{\mu}+\hat{K} \mu\right]
$$

so that the lower bound is given by the EOQ if $\mu \leq \sqrt{2 / \hat{K}}$.

As a numerical illustration, suppose that there is a single supplier with an exponentially distributed random capacity. The numerical results are given in Table 2 for a selected set of values. The table also includes previously developed bounds $[L, U]$ where $L=\max \{\sqrt{2 K D / h}, K D \mu / h\}, U=(1 / \mu)+$ $K D \mu / h$ and the EOQ $(\sqrt{2 K D / h})$.

One can observe the sensitivity of the optimal solution on the model parameters. The optimal order quantity $Q$ increases as the order cost $K$ or demand $D$ or parameter $\mu$ increases or the holding cost $h$ decreases. Note that the cycle length $E[T]$ does not necessarily increase with $Q$ as in the deterministic EOQ model. Due to the randomness of the capacity, it also depends on how $\mu$ or $D$ varies in addition to the change in $Q$. The approximations provided by (26) are quite good since the interval $[L, U]$ is short for large $\mu$ as in case 12 . For small values of $\mu$, the EOQ $(\sqrt{2 K D / h})$ may provide a good approximation as in case 1 depending on the value of $K D / h$. The approximation will be better for smaller values of this ratio. 
Table 2. Numerical results of the single supplier problem with exponential capacity.

\begin{tabular}{rrrrrrrrrr}
\hline No. & $K$ & $D$ & $h$ & $\mu$ & $Q$ & $T C(Q)$ & $100 E[T]$ & {$[L, U]$} & EOQ \\
\hline 1 & 1 & 100 & 3 & 0.025 & 8.5 & 25.4 & 7.6 & {$[8.2,40.8]$} & 8.2 \\
2 & 50 & 100 & 3 & 0.025 & 75.6 & 226.9 & 33.9 & {$[57.7,81.7]$} & 57.7 \\
3 & 100 & 100 & 3 & 0.025 & 121.4 & 364.2 & 38.1 & {$[83.3,123.3]$} & 81.7 \\
4 & 50 & 10 & 1 & 0.025 & 36.4 & 36.4 & 238.9 & {$[31.6,52.5]$} & 31.6 \\
5 & 50 & 20 & 1 & 0.025 & 54.9 & 54.9 & 149.2 & {$[44.7,65]$} & 44.7 \\
6 & 50 & 500 & 1 & 0.025 & 665.0 & 665.0 & 8.0 & {$[625,665]$} & 223.6 \\
7 & 50 & 100 & 0.1 & 0.025 & 1290.0 & 129.0 & 40.0 & {$[1250,1290]$} & 316.2 \\
8 & 50 & 100 & 1 & 0.025 & 164.3 & 164.3 & 39.3 & {$[125,165]$} & 100 \\
9 & 50 & 100 & 10 & 0.025 & 36.4 & 363.9 & 23.9 & {$[31.6,52.5]$} & 31.6 \\
10 & 1 & 100 & 3 & 0.25 & 12.1 & 36.4 & 3.8 & {$[8.3,12.3]$} & 8.2 \\
11 & 1 & 100 & 3 & 0.50 & 18.7 & 56.0 & 2.0 & {$[16.7,18.7]$} & 8.2 \\
12 & 1 & 100 & 3 & 1.5 & 50.7 & 152.0 & 0.7 & {$[50,50.7]$} & 8.2 \\
\hline
\end{tabular}

Now let us consider the general case where there are multiple suppliers with exponentially distributed capacities and derive some properties of the optimal solution. Before we state Theorem 5, consider

$$
f(q)=q-\frac{1}{\mu_{1}}\left(1-e^{-\mu_{1} q}\right)
$$

the difference between $q$ and $E\left[Y_{q}\right]$ for the first supplier.

THEOREM 5: Consider the EOQ model with $n$ suppliers that have exponential capacities so that $A_{i} \sim$ Exponential $\left(\mu_{i}\right)$ for some $\mu_{i}>0$ and $i=1,2, \ldots, n$. The optimal order quantities can be found as the unique nonnegative solution of the equations

$$
\begin{aligned}
& \frac{2 K_{n} D}{h}=\frac{2}{\mu_{1}^{2}}\left(\mu_{1} q_{1}+e^{-\mu_{1} q_{1}}-1\right) \\
& +\sum_{i=2}^{n} \frac{2}{\mu_{1} \mu_{i}}\left(\mu_{1} q_{1}+\left(1-e^{-\mu_{1} q_{1}}\right) W\left(-e^{-1-\mu_{i} f\left(q_{1}\right)}\right)\right) \\
& +2 \sum_{i=2}^{n-1} \sum_{j=i+1}^{n} \frac{1}{\mu_{i}} \frac{1}{\mu_{j}}\left(1+W\left(-e^{-1-\mu_{i} f\left(q_{1}\right)}\right)\right) \\
& \quad \times\left(1+W\left(-e^{-1-\mu_{j} f\left(q_{1}\right)}\right)\right)
\end{aligned}
$$

and

$$
q_{k}=f\left(q_{1}\right)+\frac{1}{\mu_{k}}\left[1+W\left(-e^{-\left(1+\mu_{k} f\left(q_{1}\right)\right)}\right)\right]
$$

for $k=2,3, \ldots, n$.

Note that when there is a single supplier, (30) is equivalent to (22). Theorem 5 gives a simple procedure to compute the optimal order quantities. First, the optimal order quantity is determined for the first supplier as the unique nonnegative solution of (30); then (31) is solved to compute the optimal order quantities for all other suppliers.

We provide a numerical illustration in Table 3 for two suppliers with the same data used for the uniform suppliers case where the results are given in Table 1. Recall that the cost parameters are $K_{2}=200, D=32$, and $h=2$ with $\mathrm{EOQ}=80$. We choose the parameters of the exponential distribution so that means are the same when compared with the uniform case. In other words, we set $1 / \mu_{i}=a_{i} / 2$ so that $2 / \mu_{i}=a_{i}$ and one can compare the results in the two tables to see the effects of the uniform and exponential distributions. Since there are no upper bounds on the order quantities as in the uniform case, the optimal solution is determined uniquely by (30) and (31). Note also that $Q_{i}-E\left[Y_{Q_{i}}\right]$ is always the same for both suppliers, unlike the uniform case. As the mean capacity of supplier 2 is decreased by increasing $\mu_{2}$, the expected quantity $E\left[Y_{Q_{2}}\right]$ that is actually received also decreases steadily. But, note that the order quantity eventually gets close to the mean, which was also the case for the uniform distribution.

\section{DIVERSIFICATION UNDER IDENTICAL SUPPLIERS}

We now discuss the case when all supplier capacities are independent and identically distributed. The results for the general case obviously apply when the capacities are identical. Yet one cannot clearly identify the effect of the number of suppliers on the optimal diversification scheme for nonidentical suppliers, since the scheme is also dependent on the individual yield structures of different suppliers. By considering the identical suppliers case, one can isolate the effect of the number of suppliers on the optimal order policy. 
Table 3. Numerical results of the two suppliers problem with exponential capacities.

\begin{tabular}{crrccccc}
\hline $2 / \mu_{1}$ & $2 / \mu_{2}$ & $Q_{1}$ & $Q_{2}$ & $Q_{i}-E\left[Y_{Q_{i}}\right]$ & $E\left[Y_{Q_{1}}\right]$ & $E\left[Y_{Q_{2}}\right]$ & $T C$ \\
\hline 85 & 85 & 57.48 & 57.48 & 25.97 & 31.51 & 31.51 & 177.97 \\
& 50 & 70.27 & 58.50 & 35.91 & 34.37 & 22.59 & 185.73 \\
& 30 & 82.74 & 61.05 & 46.31 & 36.48 & 14.74 & 194.98 \\
& 25 & 86.82 & 62.25 & 49.83 & 36.99 & 12.41 & 198.47 \\
& 10 & 101.94 & 68.30 & 63.30 & 38.64 & 5.00 & 213.88 \\
& 5 & 108.10 & 71.44 & 68.94 & 39.16 & 2.50 & 221.19 \\
100 & 112.12 & 73.66 & 72.66 & 39.46 & 1.00 & 226.23 \\
& 100 & 54.13 & 54.13 & 21.07 & 33.07 & 33.07 & 174.39 \\
& 50 & 69.08 & 53.73 & 31.64 & 37.44 & 22.09 & 182.34 \\
& 30 & 80.20 & 54.86 & 40.25 & 39.24 & 14.61 & 189.62 \\
& 20 & 87.83 & 56.43 & 46.46 & 41.37 & 9.96 & 195.59 \\
& 10 & 97.12 & 59.29 & 54.29 & 42.83 & 5.00 & 204.24 \\
& 5 & 102.43 & 61.38 & 58.88 & 43.55 & 2.50 & 209.87 \\
& 2 & 105.86 & 62.88 & 61.88 & 43.98 & 1.00 & 213.73 \\
\hline
\end{tabular}

Since the suppliers are identical $E\left[Y_{q_{i}}\right]=E\left[Y_{q_{j}}\right]$ and $E\left[Y_{q_{i}}^{2}\right]=E\left[Y_{q_{i}}^{2}\right]$ for all $i, j$, the symmetric nature of the formulation reduces our multivariate optimization problem in (4) to one involving the single decision variable $q=q_{1}=$ $q_{2}=\cdots=q_{n}$ with average total cost

$$
\min _{q \geq 0} T C(q)=\frac{K_{n} D}{n E\left[Y_{q}\right]}+\frac{1}{2} h\left[\frac{E\left[Y_{q}^{2}\right]+(n-1) E\left[Y_{q}\right]^{2}}{E\left[Y_{q}\right]}\right] .
$$

THEOREM 6: The optimal common order quantity $Q_{n}$ of the random capacity EOQ model with $n$ identical suppliers is the unique finite solution of the equation

$$
2 n q E\left[Y_{q}\right]+n(n-1) E\left[Y_{q}\right]^{2}-n E\left[Y_{q}^{2}\right]=\frac{2 K_{n} D}{h} .
$$

Now we derive and illustrate the effects of diversification among the suppliers when they are identical with uniformly and exponentially distributed capacities, respectively. Note that the total order quantity is $n Q_{n}$ and it is an important quantity to measure the effects of diversification. We assume that $K_{n}=K$ so that the cost of ordering is a constant irrespective of the number of suppliers. However, we also illustrate the case where $K_{n}=K+n k, K, k \geq 0$.

\subsection{Suppliers with Uniform Capacities}

When $a_{k}=a$, then $q_{k}=q$ for all $k$ and the optimality condition for the identical uniform capacities problem (33) can be rewritten as

$w_{n}(q)=\left[\frac{n(n-1)}{4 a^{2}}\right] q^{4}+\left[\frac{(2-3 n) n}{3 a}\right] q^{3}+n^{2} q^{2}=\frac{2 K D}{h}$ using (12)-(14). One can show that $w_{n}(q)$ is strictly increasing on $[0, a]$ with $w_{n}(0)=0$ and $w_{n}(a)=\left(\left(3 n^{2}+8 n-\right.\right.$ 3)/12) $a^{2}$. Therefore, the optimal order quantity is the unique nonnegative solution of (34) in $[0, a]$ if $a^{2} \geq 24 K D /\left(3 n^{2}+\right.$ $8 n-3) h$. However, if $a^{2}<24 K D /\left(3 n^{2}+8 n-3\right) h$, then the optimal order quantity is $a$. Let

$$
n^{*}=\min \left\{n \geq 1: 3 n^{2}+8 n-3 \geq \frac{24 K D}{a^{2} h}\right\} ;
$$

then it follows that $Q_{n}=a$ and the total order quantity is $n Q_{n}=n a$ whenever $n<n^{*}$. This implies that the total order quantity increases on $\left[1, n^{*}\right]$ because the suppliers do not have sufficient total capacity and one should order the maximum amount possible. However, once the number of suppliers $n$ exceeds $n^{*}$, the total order quantity decreases.

COROLLARY 7: Suppose there are $n$ identical suppliers with uniformly distributed capacities so that $A_{i} \sim U[0, a]$ for some $a>0$ and all $i=1,2, \ldots n$. If $K_{n}=K$ for all $n \geq 1$, then

$$
(n+1) Q_{n+1} \leq n Q_{n}
$$

for all $n \geq n^{*}$ and the total order quantity decreases as $n$ increases. Moreover, $\lim _{n \rightarrow+\infty} n Q_{n}=\mathrm{EOQ}=\sqrt{2 K D / h}$ for any $a$.

We now provide some illustrations in Fig. 1, where a replenishment order is given simultaneously to $n$ identical suppliers, each having uniformly distributed capacities on $[0, a]$. We see that as the number of identical suppliers increases, the total optimal order quantity $n Q_{n}$ decreases and converges to the optimal order quantity of the incapacitated or certain yield model with $\mathrm{EOQ}=\sqrt{2 K D / h}=80$. In other words, if the number of suppliers is large, then there is almost 


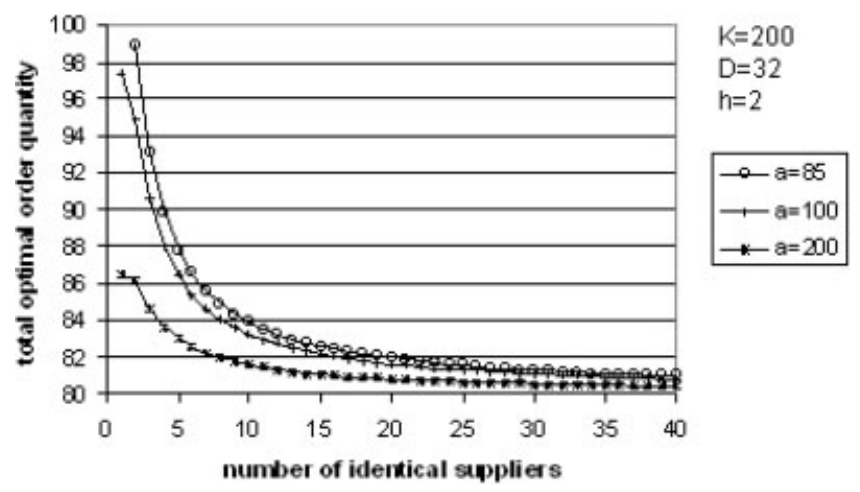

Figure 1. Total optimal order quantity versus number of identical suppliers.

no risk of getting stockout at any supplier; thus, all orders are fully received and therefore it is optimal to diversify the $\mathrm{EOQ}=80$ among $n$ suppliers.

It is obvious that diversification decreases the total order quantity more significantly when the maximum capacity is closer to the $\mathrm{EOQ}=80$. If all suppliers have infinite capacities, then the yield is certain and optimal policy is to order $\mathrm{EOQ}=80$ from a single supplier. However, if the capacity is random and bounded by $a=200$, then the order quantity is $Q_{1}=86$, which is greater than the EOQ. If the capacity bound is even less than 200, say $a=100$, then the optimal order quantity increases to $Q_{1}=97$.

In Fig. 2, the effect of the diversification on the optimal expected cost is illustrated. As the number of identical suppliers increases, the optimal expected cost decreases and converges to the cost of an infinite capacity EOQ model given by $\sqrt{2 K D h}=160$. In our model, diversification with a large number of suppliers is always beneficial, allowing order quantities and expected costs to decrease since the setup cost $K=200$ is not related to the number of suppliers used.

Figure 3 depicts the behavior of the expected total yield $n E\left[Y_{Q_{n}}\right]$ as a function of the number of identical suppliers

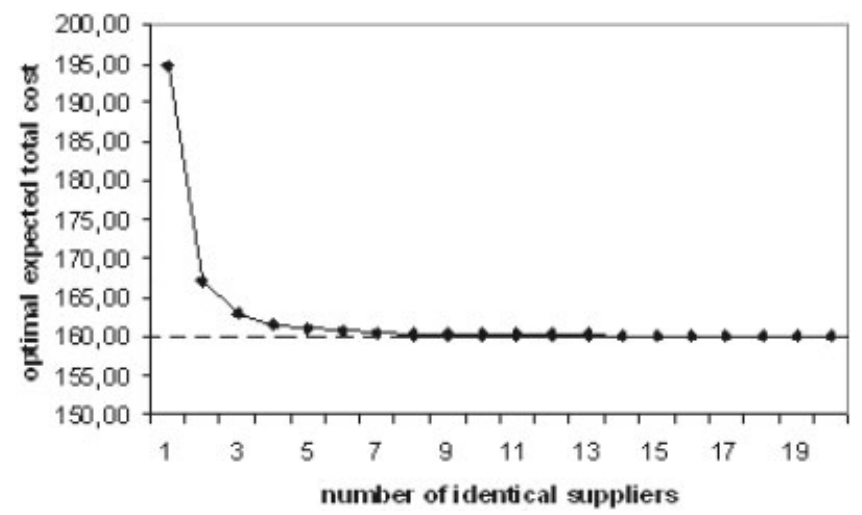

Figure 2. Optimal expected total cost versus number of identical suppliers.

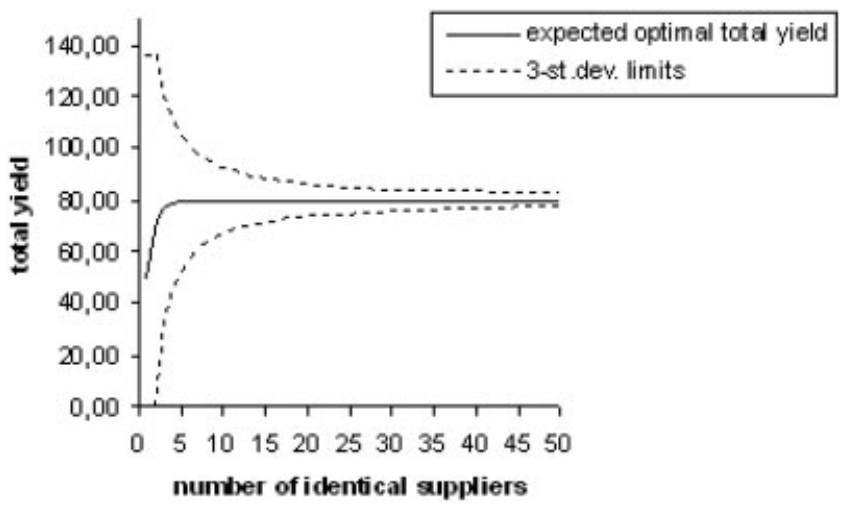

Figure 3. Total expected yield versus number of identical suppliers.

used. It is interesting to observe that as $n$ increases, the expected total yield $n E\left[Y_{Q_{n}}\right]$ increases too, although the total order quantity $n Q_{n}$ decreases. Although no formal proof is provided, we can see that it is due to the reduced probability of getting stockout at any supplier, when less is ordered from each. It follows by observing the 3 -standard deviation bounds of the total random yield that when more suppliers are used, risk is diversified and this leads to a decrease in the variability of the total yield. Figures 1 and 3 show that when the orders are diversified, less is ordered but more is expected and they both converge to the EOQ with less deviation when $n$ is very large.

\subsection{Suppliers with Exponential Capacities}

Following the similar discussion for the uniform capacities case, now we consider identical suppliers with exponential capacities and illustrate the effect of diversification.

If there are two identical suppliers, then by using (33) with $n=2$ we obtain

$$
\frac{2 \mu Q_{2}+e^{-2 \mu Q_{2}}-1}{\mu^{2}}=\frac{K_{2} D}{h}
$$

where $Q_{2}$ is the optimal order quantity for each one of the two identical suppliers. Now, the solution is

$$
Q_{2}=\frac{1}{2 \mu}\left[1+\hat{K}_{2} \mu^{2}+W\left(-e^{-\left(1+\hat{K}_{2} \mu^{2}\right)}\right)\right]
$$

where $\hat{K}_{2}=K_{2} D / h$.

The comparison of (23) and (38) indicates that if $K_{2}=$ $K_{1}=K$ so that $\hat{K}_{2}=\hat{K}_{1}$, then $2 Q_{2}=Q_{1}$. This is an amazing result, which states that the total order quantity is the same for the single and double supplier models. The ordering cost is the same in both cases and the order is diversified between the two suppliers by distributing the order quantity equally among them. However, if $K_{2} \geq K_{1}$, then one can 
easily verify that $2 Q_{2} \geq Q_{1}$ by using the fact that $W$ is increasing on $\left[-e^{-1}, 0\right]$.

For arbitrary $n \geq 1$, the optimality condition (33) yields the unique solution of $Q_{n}$ as

$$
\begin{array}{r}
\frac{\mu n Q_{n}-n(n-2) e^{-\mu Q_{n}}+0.5 n(n-1) e^{-2 \mu Q_{n}}}{\mu^{2}}+0.5 n(n-3) \\
=\frac{K_{n} D}{h} .
\end{array}
$$

COROLLARY 8: Suppose there are $n$ identical suppliers having exponentially distributed capacities with parameter $\mu>0$. If $K_{n}=K$ for all $n \geq 1$, then

$$
(n+1) Q_{n+1} \leq n Q_{n}
$$

so that the total order quantity decreases as $n$ increases. Moreover, $\lim _{n \rightarrow+\infty} n Q_{n}=\mathrm{EOQ}=\sqrt{2 K D / h}$ for any $\mu$.

Corollary 8 implies that, if the fixed ordering cost does not increase, then the total order quantity decreases with the number of suppliers. Apparently, diversification of suppliers decreases the risk of having insufficient stock at a single supplier.

\subsection{How Many Suppliers?}

A very interesting and relevant issue in a retailer's diversification among suppliers is the selection of the suppliers to work with. One would need a model that allows different unit prices for different suppliers in order to discriminate among suppliers. But one can still address the issue of how many suppliers a retailer should work with within the confines of our model. In this section we assume that all suppliers are identical so that we can isolate the effect of number of suppliers used.

We assume that the ordering cost, $K_{n}$, changes with the number of suppliers, $n$, in the form of $K+k n$ where $K$ is the fixed ordering cost and there is a variable cost of ordering, $k$, $k>0$ for each supplier used. We have already shown that the total cost decreases with the number of suppliers used when the ordering cost is independent of the number of suppliers used. Thus, it is optimal to use all available suppliers. However, we now show that if the ordering cost increases linearly with the number of suppliers, there is an optimal number of suppliers that may be less than the number of available suppliers.

We consider $n$ identical suppliers having exponentially distributed capacities with equal parameter $\mu$. Letting $K_{n}=$ $K+k n$ in (39), the optimal order quantity, $q_{n}$, is found and the expected total cost is plotted for different levels of $n$ and $\mu$ where $D=32, K=180, k=10, h=2$. It is observed from Fig. 4 that the benefit of increasing the number of suppliers is large initially, but eventually decreases and then turns

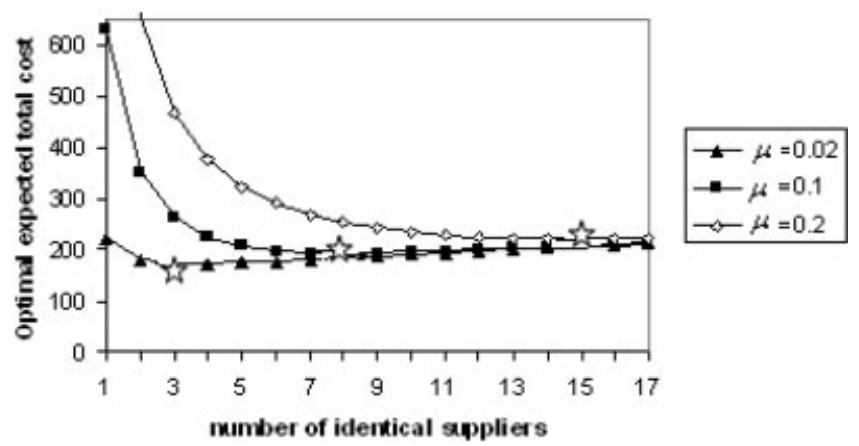

Figure 4. Expected total cost versus number of identical suppliers.

negative, in agreement with intuition. This implies that there exists an optimal number of suppliers. It is clear that when the capacity parameter, $\mu$ increases (i.e., the expected capacity, $1 / \mu$, decreases) and the optimal number of suppliers to work with increases in order to reduce the stockout risk at any supplier. Moreover, the optimal expected total cost is greater when the expected capacities are lower (i.e., $\mu$ is greater).

\section{CONCLUSION}

In this study, our objective is to introduce an EOQ model with multiple suppliers and random capacities that enables us to obtain intuition for ordering policy decisions. It is remarkable to see that when the optimal policy is applied, the expected number of unfulfilled order units from all suppliers must all be the same. Noting that this property applies only for the unbounded random capacity problem, the model with bounded capacities is analyzed under the special case of uniformly distributed capacities. If the solution of the first order condition violates the capacity constraint for any supplier, then it is optimal to order at the capacity for that supplier and for all suppliers with lower capacity bounds. Moreover, as an illustration of the unbounded capacity case, the exponential capacities problem is analyzed and a characterization for the optimal order quantities is obtained. Then the results for the two cases are compared and contrasted. Finally the effect of diversification is discussed under the settings of identical suppliers with uniformly distributed capacities and exponentially distributed capacities to show that the total optimal order quantity decreases and converges to $E O Q$ as the number of suppliers increase. Furthermore, when the ordering cost increases linearly with the number of suppliers, there is an optimal level for the number of suppliers used.

Our results indicate that computing the optimal order quantities is not very difficult, especially when compared with the complexity of the problem. Given the stochastic structure of the random capacity, one can easily compute this quantity to almost arbitrary precision. However, we also like to point out that much needs to be done in improving the model. The 
obvious generalization would be to have suppliers with different ordering and holding costs. This presents a challenge in modeling as well as in the optimization. Characterizations on the optimal order quantities under this setting would be very helpful to the practitioner, especially if they present further intuition into the decision-making process about how many and which suppliers to use. Finally, computational difficulties when the cost function is more complex will need to be resolved.

\section{APPENDIX}

PROOF (THEOREM 1): We check the first order optimality conditions to find the optimal order quantity that minimizes $T C\left(q_{1}, q_{2}, \ldots, q_{n}\right)$. Differentiating (4) with respect to $q_{k}$ gives

$$
\begin{array}{r}
\frac{\partial T C\left(q_{1}, q_{2}, \ldots, q_{n}\right)}{\partial q_{k}}=\frac{\bar{F}_{k}\left(q_{k}\right)}{2\left(\sum_{i=1}^{n} E\left[Y_{q_{i}}\right)^{2}\right.} . \\
{\left[-2 K_{n} D+h\left(2\left(q_{k}+\sum_{i \neq k} E\left[Y_{q_{i}}\right]\right) \sum_{i=1}^{n} E\left[Y_{q_{i}}\right]-\sum_{i=1}^{n} E\left[Y_{q_{i}}^{2}\right]\right.\right.} \\
\left.\left.-2 \sum_{i=1}^{n-1} \sum_{j=i+1}^{n} E\left[Y_{q_{i}}\right] E\left[Y_{q_{j}}\right]\right)\right] .
\end{array}
$$

Note that the denominator is always nonnegative and since $\bar{F}_{k}\left(q_{k}\right)>0$ for all $q_{k} \geq 0$, the second term of the numerator should be zero at the optimal solution so that

$$
\begin{aligned}
2\left(q_{k}+\sum_{i \neq k} E\left[Y_{q_{i}}\right]\right) \sum_{i=1}^{n} E\left[Y_{q_{i}}\right]-\sum_{i=1}^{n} E\left[Y_{q_{i}}^{2}\right] \\
-2 \sum_{i=1}^{n-1} \sum_{j=i+1}^{n} E\left[Y_{q_{i}}\right] E\left[Y_{q_{j}}\right]=\frac{2 K_{n} D}{h} .
\end{aligned}
$$

This gives us (10) by taking $k=1$ and canceling the common terms. Note that we have $n$ equations in the form of (42) where the right-hand side is the constant $2 K_{n} D / h$, which is in fact equal to $\mathrm{EOQ}^{2}$. Thus, by equating these equations and canceling the common terms, (11) follows directly.

We now show that the solution $\left\{q_{i}^{0}>0 ; i=1,2, \ldots, n\right\}$ satisfying the first order conditions defined by (10) and (11) is unique. By (11) we can see that for any fixed quantity $q_{1}$, the other order quantities are uniquely determined since $q_{i}-E\left[Y_{q_{i}}\right]$ is zero when $q_{i}=0$ and it is an increasing function of $q_{i}$. Yet one can still freely choose any $q_{1}$ and get other order quantities such that (11) is still satisfied. Thus, we need to show that only one of these infinitely many solutions also satisfies (10). Using (6), the implicit differentiation of (11) yields

$$
d q_{1}-\bar{F}_{1}\left(q_{1}\right) d q_{1}=d q_{2}-\bar{F}_{2}\left(q_{2}\right) d q_{2}=\cdots=d q_{n}-\bar{F}_{n}\left(q_{n}\right) d q_{n} .
$$

Since $1-\bar{F}_{i}\left(q_{i}\right)=F_{i}\left(q_{i}\right)$, we have the relation

$$
\frac{d q_{i}}{d q_{1}}=\frac{F_{1}\left(q_{1}\right)}{F_{i}\left(q_{i}\right)}
$$

for all $i, k$. Note that by using (11), one can express all optimal order quantities as a function of $q_{1}$. So, we define the left-hand side of (10) as $w\left(q_{1}, q_{2}\left(q_{1}\right), \ldots, q_{n}\left(q_{1}\right)\right)$. Obviously, $w(0,0, \ldots, 0)=0$, so if we can show that $w$ is increasing for $q_{1}>0$, then the solution satisfying (10) is unique since the right-hand side is a positive constant given by $\mathrm{EOQ}^{2}$.
Using (6) and (9), the derivative $d w\left(q_{1}, q_{2}\left(q_{1}\right), \ldots, q_{n}\left(q_{1}\right)\right) / d q_{1}$ can be obtained as

$$
\begin{aligned}
2\left[1+\sum_{i \neq 1} \bar{F}_{i}\left(q_{i}\right) \frac{d q_{i}}{d q_{1}}\right] \sum_{i=1}^{n} E\left[Y_{q_{i}}\right] \\
+2\left(q_{1}+\sum_{i \neq 1} E\left[Y_{q_{i}}\right]\right)\left(\sum_{i=1}^{n} \bar{F}_{i}\left(q_{i}\right) \frac{d q_{i}}{d q_{1}}\right)-\sum_{i=1}^{n} 2 q_{i} \bar{F}_{i}\left(q_{i}\right) \frac{d q_{i}}{d q_{1}} \\
-2 \sum_{i=1}^{n-1} \sum_{j=i+1}^{n} \bar{F}_{i}\left(q_{i}\right) \frac{d q_{i}}{d q_{1}} E\left[Y_{q_{j}}\right]+E\left[Y_{q_{i}}\right] \bar{F}_{j}\left(q_{j}\right) \frac{d q_{j}}{d q_{1}} .
\end{aligned}
$$

After substituting (44) in (45), (45) becomes

$$
\begin{gathered}
2\left[1+\sum_{i \neq 1} \frac{\bar{F}_{i}\left(q_{i}\right)}{F_{i}\left(q_{i}\right)} F_{1}\left(q_{1}\right)\right] \sum_{i=1}^{n} E\left[Y_{q_{i}}\right]+2\left[q_{1}+\sum_{i \neq 1} E\left[Y_{q_{i}}\right]\right] \\
\times \sum_{i=1}^{n} \frac{\bar{F}_{i}\left(q_{i}\right)}{F_{i}\left(q_{i}\right)} F_{1}\left(q_{1}\right)-\sum_{i=1}^{n} 2 q_{i} \frac{\bar{F}_{i}\left(q_{i}\right)}{F_{i}\left(q_{i}\right)} F_{1}\left(q_{1}\right) \\
-2 \sum_{i=1}^{n-1} \sum_{j=i+1}^{n}\left[\frac{\bar{F}_{i}\left(q_{i}\right)}{F_{i}\left(q_{i}\right)} F_{1}\left(q_{1}\right) E\left[Y_{q_{j}}\right]+\frac{\bar{F}_{j}\left(q_{j}\right)}{F_{j}\left(q_{j}\right)} F_{1}\left(q_{1}\right) E\left[Y_{q_{i}}\right]\right] .
\end{gathered}
$$

Notice that the last term in (46) can be written as

$$
\begin{array}{r}
\sum_{i=1}^{n-1} \sum_{j=i+1}^{n}\left[\frac{\bar{F}_{i}\left(q_{i}\right)}{F_{i}\left(q_{i}\right)} F_{1}\left(q_{1}\right) E\left[Y_{q_{j}}\right]+\frac{\bar{F}_{j}\left(q_{j}\right)}{F_{j}\left(q_{j}\right)} F_{1}\left(q_{1}\right) E\left[Y_{q_{i}}\right]\right] \\
=\sum_{i=1}^{n} \sum_{j=1, j \neq i}^{n}\left(\frac{\bar{F}_{i}\left(q_{i}\right)}{F_{i}\left(q_{i}\right)} F_{1}\left(q_{1}\right) E\left[Y_{q_{j}}\right]\right) . \\
=F_{1}\left(q_{1}\right)\left[\sum_{i=1}^{n} \sum_{j=1}^{n} \frac{\bar{F}_{i}\left(q_{i}\right)}{F_{i}\left(q_{i}\right)} E\left[Y_{q_{j}}\right]-\sum_{i=1}^{n} \frac{\bar{F}_{i}\left(q_{i}\right)}{F_{i}\left(q_{i}\right)} E\left[Y_{q_{i}}\right]\right] \\
=F_{1}\left(q_{1}\right) \sum_{i=1}^{n} \frac{\bar{F}_{i}\left(q_{i}\right)}{F_{i}\left(q_{i}\right)} \sum_{j=1}^{n} E\left[Y_{q_{j}}\right]-F_{1}\left(q_{1}\right) \sum_{i=1}^{n} \frac{\bar{F}_{i}\left(q_{i}\right)}{F_{i}\left(q_{i}\right)} E\left[Y_{q_{i}}\right] .
\end{array}
$$

By substituting (47) in (46) and by making further simplifications, we get

$$
\begin{aligned}
2 \sum_{i=1}^{n} E\left[Y_{q_{i}}\right] & +2 F_{1}\left(q_{1}\right) \sum_{i=1}^{n} E\left[Y_{q_{i}}\right] \sum_{i \neq 1} \frac{\bar{F}_{i}\left(q_{i}\right)}{F_{i}\left(q_{i}\right)}+2 q_{1} F_{1}\left(q_{1}\right) \sum_{i=1}^{n} \frac{\bar{F}_{i}\left(q_{i}\right)}{F_{i}\left(q_{i}\right)} \\
& +2 F_{1}\left(q_{1}\right) \sum_{i \neq 1} E\left[Y_{q_{i}}\right] \sum_{i=1}^{n} \frac{\bar{F}_{i}\left(q_{i}\right)}{F_{i}\left(q_{i}\right)}-2 F_{1}\left(q_{1}\right) \sum_{i=1}^{n} q_{i} \frac{\bar{F}_{i}\left(q_{i}\right)}{F_{i}\left(q_{i}\right)} \\
& \left.-2 F_{1}\left(q_{1}\right) \sum_{i=1}^{n} \frac{\bar{F}_{i}\left(q_{i}\right)}{F_{i}\left(q_{i}\right)} \sum_{j=1}^{n} E\left[Y_{q_{j}}\right]+2 F_{1}\left(q_{1}\right) \sum_{i=1}^{n} \frac{\bar{F}_{i}\left(q_{i}\right)}{F_{i}\left(q_{i}\right)} E\left[Y_{q_{i}}\right]\right],
\end{aligned}
$$

which can be written as

$$
\begin{aligned}
& 2 \sum_{i=1}^{n} E\left[Y_{q_{i}}\right]-2 \bar{F}_{1}\left(q_{1}\right) \sum_{i=1}^{n} E\left[Y_{q_{i}}\right]+2 q_{1} F_{1}\left(q_{1}\right) \sum_{i=1}^{n} \frac{\bar{F}_{i}\left(q_{i}\right)}{F_{i}\left(q_{i}\right)} \\
&+2 F_{1}\left(q_{1}\right) \sum_{i \neq 1} E\left[Y_{q_{i}}\right] \sum_{i=1}^{n} \frac{\bar{F}_{i}\left(q_{i}\right)}{F_{i}\left(q_{i}\right)}-2 F_{1}\left(q_{1}\right) \sum_{i=1}^{n} q_{i} \frac{\bar{F}_{i}\left(q_{i}\right)}{F_{i}\left(q_{i}\right)} \\
&+2 F_{1}\left(q_{1}\right) \sum_{i=1}^{n} \frac{\bar{F}_{i}\left(q_{i}\right)}{F_{i}\left(q_{i}\right)} E\left[Y_{q_{i}}\right] .
\end{aligned}
$$


Rewriting the fourth term in (49) and reorganizing the terms gives

$$
\begin{aligned}
& 2 F_{1}\left(q_{1}\right) \sum_{i=1}^{n} E\left[Y_{q_{i}}\right]+2 q_{1} F_{1}\left(q_{1}\right) \sum_{i=1}^{n} \frac{\bar{F}_{i}\left(q_{i}\right)}{F_{i}\left(q_{i}\right)} \\
&+2 F_{1}\left(q_{1}\right)\left(\sum_{i=1}^{n} E\left[Y_{q_{i}}\right] \sum_{i=1}^{n} \frac{\bar{F}_{i}\left(q_{i}\right)}{F_{i}\left(q_{i}\right)}-E\left[Y_{q_{1}}\right] \sum_{i=1}^{n} \frac{\bar{F}_{i}\left(q_{i}\right)}{F_{i}\left(q_{i}\right)}\right) \\
&-2 F_{1}\left(q_{1}\right) \sum_{i=1}^{n} \frac{\bar{F}_{i}\left(q_{i}\right)}{F_{i}\left(q_{i}\right)}\left(q_{i}-E\left[q_{i}\right]\right),
\end{aligned}
$$

which is equal to

$$
\begin{array}{r}
2 F_{1}\left(q_{1}\right)\left\{\sum_{i=1}^{n} E\left[Y_{q_{i}}\right]+\sum_{i=1}^{n} \frac{\bar{F}_{i}\left(q_{i}\right)}{F_{i}\left(q_{i}\right)}\left[\left(q_{1}-E\left[Y_{q_{1}}\right]\right)-\left(q_{i}-E\left[q_{i}\right]\right)\right]\right. \\
\left.+\sum_{i=1}^{n} E\left[Y_{q_{i}}\right] \sum_{i=1}^{n} \frac{\bar{F}_{i}\left(q_{i}\right)}{F_{i}\left(q_{i}\right)}\right\} .
\end{array}
$$

Since $\left(q_{1}-E\left[Y_{q_{1}}\right]\right)=\left(q_{i}-E\left[q_{i}\right]\right)$ by the first order conditions defined in (11), (51) is finally simplified as

$$
2 F_{1}\left(q_{1}\right) \sum_{i=1}^{n} E\left[Y_{q_{i}}\right]\left(1+\sum_{i=1}^{n} \frac{\bar{F}_{i}\left(q_{i}\right)}{F_{i}\left(q_{i}\right)}\right),
$$

which is definitely positive for $q_{1}>0$. So, $w\left(q_{1}, q_{2}\left(q_{1}\right), \ldots, q_{n}\left(q_{1}\right)\right)$ is an increasing function of $q_{1}$, and since $w(0,0, \ldots, 0)=0$, there is a unique solution in the form $\left[q_{1}^{0}, q_{2}^{0}, \ldots, q_{n}^{0}\right]$ satisfying the first order conditions defined by (10) and (11).

In order to determine the nature of the critical point, we need to check the Hessian of $T C\left(q_{1}, q_{2}, \ldots, q_{n}\right)$ at this point. The second degree partial derivatives of the function at the critical point must be computed. Taking the derivative of (41) with respect to $q_{k}$ and using the fact that (41) is equal to zero at the critical point, we obtain

$$
\begin{gathered}
\left.\frac{\partial^{2} T C\left(q_{1}, q_{2}, \ldots, q_{n}\right)}{\partial q_{k}^{2}}\right|_{\left(q_{1}^{0}, q_{2}^{0}, \ldots, q_{n}^{0}\right)}=\frac{h \bar{F}_{k}\left(q_{k}^{0}\right)}{2\left(\sum_{i=1}^{n} E\left[Y_{q_{i}^{0}}\right]\right)^{2}} \\
\times\left[\begin{array}{c}
\left.2 \sum_{i=1}^{n} E\left[Y_{q_{i}^{0}}\right]+2\left(q_{k}^{0}+\sum_{i \neq k} E\left[Y_{q_{i}^{0}}\right]\right) \bar{F}_{k}\left(q_{k}^{0}\right)-2 q_{k}^{0} \bar{F}_{k}\left(q_{k}^{0}\right)\right] \\
-2 \bar{F}_{k}\left(q_{k}^{0}\right) \sum_{i \neq k} E\left[Y_{q_{i}^{0}}\right]
\end{array}\right] \\
=\frac{h \bar{F}_{k}\left(q_{k}^{0}\right)}{\sum_{i=1}^{n} E\left[Y_{q_{i}^{0}}\right]}>0 .
\end{gathered}
$$

Taking the derivative of (41) with respect to $q_{j}$ and, again, using the fact that (41) is equal to zero at the critical point, we obtain

$$
\begin{gathered}
\left.\frac{\partial^{2} T C\left(q_{1}, q_{2}, \ldots, q_{n}\right)}{\partial q_{k} \partial q_{j}}\right|_{\left(q_{1}^{0}, q_{2}^{0}, \ldots, q_{n}^{0}\right)}=\frac{h \bar{F}_{k}\left(q_{k}^{0}\right)}{2\left(\sum_{i=1}^{n} E\left[Y_{q_{i}^{0}}\right]\right)^{2}} \\
\times\left[\begin{array}{c}
2 \bar{F}_{j}\left(q_{j}^{0}\right) \sum_{i=1}^{n} E\left[Y_{q_{i}^{0}}\right]+2\left(q_{k}^{0}+\sum_{i \neq k} E\left[Y_{q_{i}^{0}}\right]\right) \bar{F}_{j}\left(q_{j}^{0}\right)-2 q_{j}^{0} \bar{F}_{j}\left(q_{j}^{0}\right) \\
-2 \bar{F}_{j}\left(q_{j}^{o}\right) \sum_{i \neq j} E\left[Y_{q_{i}^{o}}\right]
\end{array}\right] \\
=\frac{h\left(\bar{F}_{k}\left(q_{k}^{0}\right)\right)^{2}}{2\left(\sum_{i=1}^{n} E\left[Y_{q_{i}^{0}}\right]\right)^{2}}\left[\left(q_{k}^{0}-E\left[Y_{q_{k}^{0}}\right]\right)-\left(q_{j}^{0}-E\left[Y_{q_{j}^{0}}\right]\right)\right]
\end{gathered}
$$

Since $\left(q_{k}-E\left[Y_{q_{k}}\right]\right)=\left(q_{j}-E\left[Y_{q_{j}}\right]\right)$ at the critical point by the first order conditions given in (11), (54) is equal to zero. Hence, all the off-diagonal entries of the Hessian are 0, while all the diagonal entries are positive. This means that the Hessian is a positive definite matrix and the unique critical point defined by (10) and (11) is a local minimum. Moreover, the partial derivatives of the total cost function indicate that at any boundary (i.e., where $q_{i}=0$ ), the total cost function decreases as one gets away from the boundary (i.e., as $q_{i}$ increases). Thereby, the minimum must occur inside the feasible region. Thus, the critical point is the global minimum for the $T C\left(q_{1}, q_{2}, \ldots, q_{n}\right)$ function.

PROOF (THEOREM 3): Since $A_{i} \backsim$ Uniform $\left[0, a_{i}\right], E\left[Y_{q_{i}}\right]$ and $E\left[Y_{q_{i}}^{2}\right]$ can be written as in (12) and (13). Now, consider the first order conditions given in Theorem 1. By using (12) and (13), (11) gives the first order optimality condition in (16). By further substitution, we rewrite $E\left[Y_{q_{i}}\right]$ and $E\left[Y_{q_{i}}^{2}\right]$ as

$$
\begin{gathered}
E\left[Y_{q_{i}}\right]=\sqrt{\frac{a_{i}}{a_{1}}} q_{1}-\frac{q_{1}^{2}}{2 a_{1}} \\
E\left[Y_{q_{i}}^{2}\right]=\frac{a_{i}}{a_{1}} q_{1}^{2}-\frac{2}{3 a_{1}} \sqrt{\frac{a_{i}}{a_{1}}} q_{1}^{3} .
\end{gathered}
$$

Now, we substitute $E\left[Y_{q_{i}}\right]$ and $E\left[Y_{q_{i}}^{2}\right]$ in (10) to derive the first order optimality condition in (15). This leads to

$$
\begin{array}{r}
\frac{2 K_{n} D}{h}=2 q_{1} \sum_{i=1}^{n}\left(\sqrt{\frac{a_{i}}{a_{1}}} q_{1}-\frac{q_{1}^{2}}{2 a_{1}}\right)+2 \sum_{i=2}^{n} \sum_{j=i}^{n}\left(\sqrt{\frac{a_{i}}{a_{1}}} q_{1}-\frac{q_{1}^{2}}{2 a_{1}}\right) \\
\times\left(\sqrt{\frac{a_{j}}{a_{1}}} q_{1}-\frac{q_{1}^{2}}{2 a_{1}}\right)-\sum_{i=1}^{n}\left(\frac{a_{i}}{a_{1}} q_{1}^{2}-\frac{2}{3 a_{1}} \sqrt{\frac{a_{i}}{a_{1}}} q_{1}^{3}\right) \\
=\left[\frac{n(n-1)}{4 a_{1}^{2}}\right] q_{1}^{4}+\left[\frac{2-3 n}{3 a_{1}} \sum_{i=1}^{n} \sqrt{\frac{a_{i}}{a_{1}}}\right] q_{1}^{3} \\
+\left[2 \sum_{i=1}^{n} \sum_{j=i}^{n} \sqrt{\frac{a_{i}}{a_{1}}} \frac{a_{j}}{a_{1}}-\sum_{i=1}^{n} \frac{a_{i}}{a_{1}}\right] q_{1}^{2},
\end{array}
$$

where we skip the mathematical manipulations in between. Finally, let $q_{1}^{0}, q_{2}^{0}, \ldots, q_{n}^{0}$ be the solution satisfying the first order conditions in (15) and (16). If for all $i, q_{i}^{0} \leq a_{i}$, then this solution is feasible and it is the unique optimal solution by Theorem 1 .

PROOF (THEOREM 5): Since $A_{i} \backsim$ Exponential $\left(\mu_{i}\right), \quad E\left[Y_{q_{i}}\right]$ and $E\left[Y_{q_{i}}^{2}\right]$ can be written as in (20) and (21). Now, consider the optimality conditions given in Theorem 1. By using (20) and (21), (11) can be rewritten as

$$
q_{1}-\frac{1}{\mu_{1}}\left(1-e^{-\mu_{1} q_{1}}\right)=q_{k}-\frac{1}{\mu_{k}}\left(1-e^{-\mu_{k} q_{k}}\right)
$$

for any $k$. The solution of (58) gives (31) by using the Lambert $W$ function. We substitute $E\left[Y_{q_{i}}\right]$ and $E\left[Y_{q_{i}}^{2}\right]$ in (10) to derive the first order optimality 
condition in (30). This leads to

$$
\begin{aligned}
& \frac{2 K_{n} D}{h}=2 q_{1} \sum_{i=1}^{n}\left(\frac{1}{\mu_{i}}\left(1+W\left(-e^{-1-\mu_{i} f\left(q_{1}\right)}\right)\right)\right) \\
& +2 \sum_{i=2}^{n} \sum_{j=i}^{n}\left(\frac{1}{\mu_{i}}\left(1+W\left(-e^{-1-\mu_{i} f\left(q_{1}\right)}\right)\right)\right) \\
& \quad \times\left(\frac{1}{\mu_{j}}\left(1+W\left(-e^{-1-\mu_{j} f\left(q_{1}\right)}\right)\right)\right) \\
& -\sum_{i=1}^{n}\left(\frac { 2 } { \mu _ { i } ^ { 2 } } \left(1+\left(2+\mu_{i} f\left(q_{1}\right)+W\left(-e^{-1-\mu_{i} f\left(q_{1}\right)}\right)\right)\right.\right. \\
& \left.\left.\times W\left(-e^{-1-\mu_{i} f\left(q_{1}\right)}\right)\right)\right)
\end{aligned}
$$

which, after some tedious mathematical manipulations, can be shown to equal (30).

PROOF (THEOREM 6): We check the first and second order optimality conditions to find the optimal order quantity that minimizes $T C(q)$. Differentiating (32) with respect to $q$ gives

$$
\begin{aligned}
\frac{d T C(q)}{d q}=\frac{h \bar{F}(q)}{n^{2} E\left[Y_{q}\right]^{2}}\left[\left(n q E\left[Y_{q}\right]\right.\right. & -\frac{n}{2} E\left[Y_{q}^{2}\right] \\
& \left.\left.+\frac{n(n-1)}{2} E\left[Y_{q}\right]^{2}\right)-\frac{K_{n} D}{h}\right] .
\end{aligned}
$$

Note that the denominator is always nonnegative and since $\bar{F}(q)>0$ for all $q \geq 0$, the second term of the numerator should be zero at the optimal solution, which gives us the required equality in (33). Let us denote the left-hand side of (33) as

$$
w(q)=2 n q E\left[Y_{q}\right]+n(n-1) E\left[Y_{q}\right]^{2}-n E\left[Y_{q}^{2}\right] .
$$

It is observed that $w(0)=0$ and $w(q)$ is strictly increasing since $w^{\prime}(q)=$ $2 n E\left[Y_{q}\right]+2 n(n-1) E\left[Y_{q}\right] \bar{F}(q)>0$ for $q>0$. Therefore, there is a unique and finite solution satisfying $w\left(Q_{n}\right)=2 K_{n} D / h$. The fact that this is the global minimum follows by noting that $T C$ is unimodally decreasing on $\left[0, Q_{n}\right]$ and increasing on $\left[Q_{n},+\infty\right]$. This is a direct consequence of the fact that $w(q)$ is strictly increasing so that the sign of the derivative of $T C$ in (59) is the same as that of $w(q)-\left(2 K_{n} D / h\right)$.

PROOF (COROLLARY 7): Assume $n \geq n^{*}$ so that (34) is satisfied uniquely on $[0, a]$ by the optimal order quantity. We can rewrite (36) as

$$
\frac{n+1}{n} Q_{n+1} \leq Q_{n},
$$

where $w_{n}\left(Q_{n}\right)=w_{n+1}\left(Q_{n+1}\right)=2 K D / h$. Since $w_{n}(q)$ is increasing on $[0, a]$, it suffices to show that $w_{n}\left(\frac{n+1}{n} Q_{n+1}\right) \leq w_{n}\left(Q_{n}\right)=2 K D / h$ to prove (36). We now evaluate $w_{n}(q)$ for $q=\frac{n+1}{n} Q_{n+1}$ and verify that, in fact,

$$
\begin{aligned}
& w_{n}\left(\frac{n+1}{n} Q_{n+1}\right)=\left[\frac{(n-1)}{4 a^{2}}\right] \frac{(n+1)^{4}}{n^{3}} Q_{n+1}^{4} \\
&+\left[\frac{(2-3 n)}{3 a}\right] \frac{(n+1)^{3}}{n^{2}} Q_{n+1}^{3}+(n+1)^{2} Q_{n+1}^{2} \leq \frac{2 K D}{h} \\
&=w_{n+1}\left(Q_{n+1}\right) .
\end{aligned}
$$

After writing the expression (34) for $Q_{n+1}$ on the right-hand side of (62), the required inequality in (62) is true if and only if

$$
\begin{aligned}
& {\left[\frac{(n-1)(n+1)^{4}}{4 a^{2} n^{3}}-\frac{(n+1) n}{4 a^{2}}\right] Q_{n+1}^{4}} \\
& \quad+\left[\frac{(2-3 n)(n+1)^{3}}{3 a n^{2}}+\frac{(3 n+1)(n+1)}{3 a}\right] Q_{n+1}^{3} \leq 0 .
\end{aligned}
$$

In order to check whether (63) holds, we define a function $l(q)$ on $[0, a]$ so that $l\left(Q_{n+1}\right)$ is given by the left-hand side of (63). We will show more generally that $l(q) \leq 0$ for all $0 \leq q \leq a$. Note that $l(0)=0$ and

$$
\frac{d l(q)}{d q}=\frac{(n+1)}{a} q^{2}\left[\frac{q}{a}\left(2-\frac{2}{n^{2}}-\frac{1}{n^{3}}\right)+\left(-3+\frac{1}{n}+\frac{2}{n^{2}}\right)\right] .
$$

In this expression, the first and second multiplicands are always nonnegative and the third multiplicand is nonpositive for $0 \leq q \leq a$. Hence, $d l(q) / d q$ $\leq 0$ on $[0, a]$ and $l(Q) \leq 0$ for $0 \leq q \leq a$.

To prove that the total order quantity decreases to the EOQ, take $x_{n}=n Q_{n}$ and let $x=\lim _{n \rightarrow+\infty} x_{n}$ be the limit of this decreasing sequence (for $\left.n \geq n^{*}\right)$. Defining

$$
\begin{aligned}
b_{n}= & {\left[\frac{n(n-1)}{4 a^{2}}\right] Q_{n}^{4}+\left[\frac{(2-3 n) n}{3 a}\right] Q_{n}^{3}+n^{2} Q_{n}^{2} } \\
& =\left[\frac{x_{n}^{2}-\left(x_{n}^{2} / n\right) x_{n}}{4 a^{2}}\right]\left(\frac{x_{n}}{n}\right)^{2}+\frac{2 x_{n}}{3 a}\left(\frac{x_{n}}{n}\right)^{2}-\frac{x_{n}^{2}}{a}\left(\frac{x_{n}}{n}\right)+x_{n}^{2},
\end{aligned}
$$

the result follows trivially from (34) by noting that $\lim _{n \rightarrow+\infty} b_{n}=x^{2}$ for all values of $a$ so that $x=\mathrm{EOQ}=\sqrt{2 K D / h}$.

PROOF (COROLLARY 8): We have already shown that (40) holds as an equality for $n=1$. Suppose that $n \geq 2$. We can rewrite (40) as

$$
\frac{n+1}{n} Q_{n+1} \leq Q_{n}
$$

where $Q_{n}$ and $Q_{n+1}$ satisfy

$$
\mu n Q_{n}-n(n-2) e^{-\mu Q_{n}}+0.5 n(n-1) e^{-2 \mu Q_{n}}+0.5 n(n-3)=\frac{K D}{h} \mu^{2}
$$

and

$$
\begin{array}{r}
\mu(n+1) Q_{n+1}-(n+1)(n-1) e^{-\mu Q_{n+1}}+0.5(n+1) n e^{-2 \mu Q_{n+1}} \\
+0.5(n+1)(n-2)=\frac{K D}{h} \mu^{2}
\end{array}
$$

from (39). Define

$$
g(q)=\mu n q-n(n-2) e^{-\mu q}+0.5 n(n-1) e^{-2 \mu q}+0.5 n(n-3),
$$

which is clearly increasing on $[0,+\infty)$ with $g(0)=0$. So, to prove $(66)$, it suffices to show that $g\left(\frac{n+1}{n} Q_{n+1}\right) \leq g\left(Q_{n}\right)$. By (67), we know that $g\left(Q_{n}\right)=$ $K D \mu^{2} / h$ is a constant. We now evaluate $g(q)$ for $q=\frac{n+1}{n} Q_{n+1}$ and verify that in fact

$$
\begin{aligned}
& g\left(\frac{n+1}{n} Q_{n+1}\right)=\mu(n+1) Q_{n+1}-n(n-2) e^{-\mu \frac{n+1}{n} Q_{n+1}} \\
& \quad+0.5 n(n-1) e^{-2 \mu \frac{n+1}{n} Q_{n+1}}+0.5 n(n-3) \leq \frac{K D}{h} \mu^{2}=g\left(Q_{n}\right) .
\end{aligned}
$$


We insert (68) in (70) for $K D \mu^{2} / h$ so that the required inequality in (70) is true if and only if

$$
\begin{array}{r}
(n+1)(n-1) e^{-\mu Q_{n+1}}-0.5 n(n+1) e^{-2 \mu Q_{n+1}}-n(n-2) e^{-\frac{n+1}{n} \mu Q_{n+1}} \\
+0.5 n(n-1) e^{-2 \frac{n+1}{n} \mu Q_{n+1}} \leq n-1 .
\end{array}
$$

In order to check whether (71) holds, we define a function $l(q)$ on $[0,+\infty]$ so that $l\left(Q_{n+1}\right)$ is given by the left-hand side of (71). We will show more generally that $l(q) \leq n-1$ for all $q \geq 0$.

Note that $l(0)=n-1$ and $\lim _{q \rightarrow+\infty} l(q)=0 \leq n-1$. It is therefore sufficient to show that $l(q)$ is a nonincreasing function for $q \geq 0$. Thus, we differentiate $l(q)$ and check whether the derivative is nonpositive. Note that

$$
\begin{aligned}
\frac{d l(q)}{d q}=-\mu(n+1) e^{-\mu q}\left((n-1)-n e^{-\mu q}\right. & -(n-2) e^{-\mu q / n} \\
& \left.+(n-1) e^{-\mu q(n+2) / n}\right) .
\end{aligned}
$$

Apparently, (72) is nonpositive if

$$
(n-1)-n e^{-\mu q}-(n-2) e^{-\mu q / n}+(n-1) e^{-\mu q(n+2) / n} \geq 0 .
$$

Following the same argument above, we see that the left-hand side in (73) is a function of $q$ and takes values in the interval $[0, n-1]$. Thus, it is sufficient to show that (73) is nondecreasing. So, we differentiate the left-hand side of (73) with respect to $q$ and find that the derivative satisfies

$$
\left(\frac{n-2}{n}\right) \mu e^{-\mu q / n}\left[1-e^{-\mu q(n+1) / n}\right]+n \mu e^{-\mu q}\left[1-e^{-2 \mu q / n}\right] \geq 0,
$$

since both terms in (74) are nonnegative for $q \geq 0$.

To prove that the total order quantity decreases to the EOQ, take $x_{n}=$ $n Q_{n}$ and let $x=\lim _{n \rightarrow+\infty} x_{n}$ be the limit of this decreasing sequence. Defining

$$
b_{n}=\frac{\mu x_{n}-n(n-2) e^{-\left(\frac{\mu}{n}\right) x_{n}}+0.5 n(n-1) e^{-\left(\frac{2 \mu}{n}\right) x_{n}}+0.5 n(n-3)}{\mu^{2}},
$$

the result follows trivially from (39) by noting that $\lim _{n \rightarrow+\infty} b_{n}=x^{2} / 2$ for all values of $\mu$ so that $x=\mathrm{EOQ}=\sqrt{2 K D / h}$. The analysis starts by writing the exponential functions in (75) in series form using Taylor's expansion and evaluates the limit of each term to arrive at the quadratic function of $x$.

\section{ACKNOWLEDGMENTS}

This research is supported by Research Project 02N301 of Boğaziçi University, İstanbul, Turkey. The authors thank the associate editor and two anonymous referees for their detailed and insightful comments on the earlier versions of the paper.

\section{REFERENCES}

[1] R. Anupindi and R. Akella, Diversification under supply uncertainty, Manage Sci 39 (1993), 944-963.

[2] F. W. Ciarallo, R. Akella, and T. E. Morton, A periodic review production planning model with uncertain capacity and uncertain demand-optimality of extended myopic policies, Manage Sci 40 (1994), 320-332.

[3] R. M. Corless, G. H. Gonnet, D. E. G. Hare, D. J. Jeffrey, and D. E. Knuth, On the Lambert $W$ function, Adv Comput Math 5 (1996), 329-359.

[4] A. Erdem, Single-period inventory models with two vendors and random yield, Ph. D. Dissertation, Department of Industrial Engineering, Bog̃aziçi University, 1999.

[5] A. S. Erdem and S. Özekici, Inventory models with random yield in a random environment, Int J Prod Econ 78 (2002), 239-253.

[6] M. M. Fadıloğlu, E. Berk, and M. C. Gürbüz, An EOQ model with multiple vendors and binomial yield, Technical Report, Department of Industrial Engineering, Bilkent University, 2005.

[7] Y. Gerchak and M. Parlar, Yield randomness, cost tradeoffs and diversification in the EOQ model, Nav Res Logist 37 (1990), 341-354.

[8] R. Güllü, Base-stock policies for production/inventory problem with uncertain capacity levels, Eur J Oper Res 105 (1998), 43-51.

[9] Ü. Gürler and M. Parlar, An inventory problem with two randomly available suppliers, Oper Res 45 (1997), 904-918.

[10] M. Henig and Y. Gerchak, The structure of periodic review policies in the presence of random yield, Oper Res 38 (1990), 634-643.

[11] S. Karlin, One stage models with uncertainty, Studies in the Mathematical Theory of Inventory and Production, K. J. Arrow, S. Karlin, and H. Scarf (Editors), Stanford University Press, Stanford, 1958, pp. 109-134.

[12] H. L. Lee and C. A. Yano, Production control in multistage systems with variable yield losses, Oper Res 36 (1988), 269-278.

[13] S. Özekici and M. Parlar, Inventory models with unreliable suppliers in a random environment, Ann Oper Res 91 (1999), 123-136.

[14] M. Parlar and D. Berkin, Future supply uncertainty in EOQ models, Nav Res Logist 38 (1991), 107-121.

[15] M. Parlar and D. Wang, Diversification under yield randomness in inventory models, Eur J Oper Res 66 (1993), 52-64.

[16] W. Shih, Optimal inventory policies when stockouts result from defective products, Int J Prod Res 18 (1980), 677-686.

[17] E. A. Silver, Establishing the order quantity when the amount received is uncertain, INFOR 14 (1976), 32-39.

[18] Y. Wang and Y. Gerchak, Periodic review production models with variable capacity, random yield and uncertain demand, Manage Sci 42 (1996), 130-137.

[19] Y. Wang and Y. Gerchak, Continuous review inventory control when capacity is variable, Int J Prod Econ 45 (1996b), 381-388.

[20] C. A. Yano and H. L. Lee, Lot sizing with random yields: A review, Oper Res 43 (1995), 311-334. 